DOI: 10.1136/annrheumdis-2017-eular.1745

\section{THU0488 THE IMPACT OF OLIGOARTICULAR AND POLYARTICULAR JUVENILE IDIOPATHIC ARTHRITIS OVER THE FIRST FIVE YEARS FOLLOWING DIAGNOSIS}

S.J.W. Shoop-Worrall ${ }^{1,2}$, S.M. Verstappen ${ }^{1}$, J.E. McDonagh ${ }^{2,3,4}$, W. Thomson ${ }^{2,5}$, K.L. Hyrich ${ }^{1,2}$ on behalf of CAPS. ${ }^{1}$ Arthritis Research UK Centre for Epidemiology, the University of Manchester $;{ }^{2}$ NIHR Manchester Musculoskeletal BRU, Central Manchester University Hospitals NHS Foundation Trust and University of Manchester Partnership; ${ }^{3}$ Centre for MSK Research, the University of Manchester; ${ }^{4}$ Manchester Academic Health Science Centre; ${ }^{5}$ Arthritis Research UK Centre for Genetics and Genomics, the University of Manchester, Manchester, United Kingdom

Background: Information regarding longer-term outcomes in JIA largely pre-date the introduction of biologic therapies and have been cross-sectional.

Objectives: The aim of this study was to assess outcomes over the first 5 years of disease in children diagnosed with oligoarticular and polyarticular JIA since 2001.

Methods: Children with oligoarthritis, rheumatoid-factor (RF) negative or positive polyarthritis were selected if recruited to the Childhood Arthritis Prospective Study (CAPS), a UK multicentre inception cohort, between October 2001 and January 2011. The following outcomes were assessed annually to five years and included in this analysis: functional ability (Childhood Health Assessment Questionnaire (CHAQ)), the absence of limited joints, overall psychosocial health (psychosocial scale on the Child Health Questionnaire (CHQ)) and the proportion of children with $\mathrm{CHQ}$ psychosocial scores two standard deviations below the population mean $(\mathrm{CHQ}$ psychosocial $<30)$.

Outcomes were assessed descriptively over time and differences between subtypes were assessed by applying multilevel (patient-level) zero-inflated negative binomial (CHAQ), logistic (absence of limited joints, percent $\mathrm{CHQ}$ psychosocial $<30$ ) and linear (CHQ psychosocial) regression analyses, adjusting for gender, age at presentation and hospital.

Results: Of 832 children, $70 \%$ were female, $68 \%$ had oligoarticular, $28 \%$ RFnegative and $5 \%$ RF-positive polyarticular JIA. Eighty four percent had ever been treated with NSAIDs, $74 \%$ corticosteroids, $55 \%$ with DMARDs and $21 \%$ with biologics within follow-up.

Baseline CHAQ was good to moderate (median 0.8 , IQR 0.1 to 1.4 ) and only $21 \%$ of children had no limited joints reported at this time. $\mathrm{CHQ}$ psychosocial scores (median 50, IQR 39 to 55) were moderate, with $11 \%$ children scoring at least two standard deviations under the population mean. Overall improvements were evident in all outcomes over the first year then remained stable with no further improvements at the cohort level evident to five years.

Patients with RF-negative polyarthritis experienced significantly poorer outcomes across all variables than those with oligoarthritis. Those with RF-positive polyarthritis recorded similar $\mathrm{CHAQ}$ scores to patients with RF-negative polyarthritis but had the lowest odds of no limited joints (OR: $0.4,95 \% \mathrm{Cl} 0.3$ to 0.7 ) and the poorest $\mathrm{CHQ}$ psychosocial scores (4.8 points worse and 4.7 times the odds of scores $<2$ standard deviations of population mean), compared with those with oligoarthritis.

Conclusions: On average, the largest improvement in functional ability, limited joints and psychosocial health occur in the first year following diagnosis, perhaps confirming the importance of early treat-to-target strategies. Patients with polyarticular JIA subtype have poorer parent and physician-reported outcomes than those with oligoarthritis.

Disclosure of Interest: None declared

DOI: 10.1136/annrheumdis-2017-eular.3785

\section{THU0489 ADIPOSITY AND INFLAMMATORY ACTIVITY IN JUVENILE IDIOPATHIC ARTHRITIS COULD THEY BE RELATED?}

G. Diaz-Cordoves Rego ${ }^{1}$, R. Galindo-Zavala ${ }^{2}$, E. Nuñez-Cuadros ${ }^{2}$, S. Manrique-Arija ${ }^{1}$, N. Mena Vazquez ${ }^{1}$, C. Domic-Bueno ${ }^{1}$, M. Rojas-Gimenez $^{1}$ C. Fuego-Varela ${ }^{1}$, R. Caparros-Ruiz ${ }^{1}$, L. Cano-Garcia ${ }^{1}$, A. Fernandez-Nebro ${ }^{1}$. ${ }^{1}$ Rheumatology, University Regional Hospital of Malaga (HRUM). Institute Biomedical Research In Malaga (IBIMA). Malaga University. Málaga, Spain; ${ }^{2}$ Paediatric Department, Universitary Children Hospital of Malaga, Málaga, Spain

Background: Adults with rheumatoid arthritis have been shown to have a reduction in lean mass and increased adiposity, despite presenting normal Body Mass Index (BMI). Several studies have shown increased adiposity induces a proinflammatory state which lead to a worse response to treatment. There are few publications about this subject in juvenile idiopathic arthritis (JIA)

Objectives: Describing body composition and anthropometric parameters in JIA patients, and evaluating relationship between adiposity and inflammatory in these children.

Methods: Observational cross-sectional study, in JIA patients from 4 to 15 years, monitored by a Pediatric Rheumatology Unit. Monoarticular forms were excluded. Anthropometric, clinical and treatment data were recorded. DXA (measuring bone and fat mass) were obtained. Fat Mass Index (FMI) was defined as fat mass $(\mathrm{kg}) /$ height $\left(\mathrm{m}^{2}\right)$ and fat-free mass index (FFMl) as lean mass $(\mathrm{kg}) /$ height $\left(\mathrm{m}^{2}\right)$. JADAS27 index was used to evaluate inflammatoty activity.
Results: We analyzed 80 patients, whose characteristics are shown in table 1. The most frequent JIA subtype was oligoarticular (16.3\% extended, $47.5 \%$ persistent) followed by polyarticular $(25.1 \%)$. Twenty five percent of patients had uveitis. Fifty percent them had inactive disease with treatment, $26 \%$ had activity and $23 \%$ were inactive without treatment. Regarding the treatment, $52.5 \%$ were on methotrexate and $30 \%$ on a biological drug $(22.5 \%$ antiTNF $\alpha, 5 \%$ antilL-1, 2.5\% antilL-6). Disease duration average was 6.6 years $( \pm 3.7 \mathrm{SD})$. JADAS27 index mean score was $2( \pm 4 \mathrm{SD})$, CRP $4.7 \mathrm{mg} / \mathrm{l}( \pm 9,5 \mathrm{SD})$, ESR $8.7 \mathrm{~mm}$ $( \pm 7,2 \mathrm{SD})$ and $\mathrm{CHAQ} 0.17( \pm 0,38 \mathrm{SD})$. Anthropometric parameters are shown in table 1. Mean JADAS27 index score in patients with normal BMlwas lower $(1.7 \pm 3.6 \mathrm{SD})$ than mean JADAS27 index score in overweight and obese patients (3.3 $\pm 6.0 \mathrm{SD})$, although this difference was not significantly $(p=0.255)$. In multiple linear regression, an increase of 0.3 JADAS27 was observed for each unit of FMl increase $(p=0.03)$. This relationship was maintained in the multivariate analysis (B0.015; p0.01) independently of JIA subtype and received treatment.

\begin{tabular}{|c|c|}
\hline Variable & $\mathrm{n}=80$ \\
\hline Age in years, median $( \pm S D)$ & $10,7(3,3)$ \\
\hline Sex,female; n (\%) & $56(70)$ \\
\hline Race caucasian,n (\%) & $76(95)$ \\
\hline BMI Median kg/m² ( \pm SD) & $18,2(4,2)$ \\
\hline BMI Percentile, median $( \pm S \mathrm{~S})$ & $42,0(29,9)$ \\
\hline Waist perimeter, median, $\mathrm{cm}( \pm \mathrm{SD})$ & $64,8(11,3)$ \\
\hline Hip perimeter $\mathrm{cm}( \pm \mathrm{SD})$ & $77(13,6)$ \\
\hline Index waist/hip ( \pm SD) & $0,84(0,06)$ \\
\hline Obesity ${ }^{\star}, \mathrm{n}(\%)$ & $6(7,5)$ \\
\hline Overweigh ${ }^{*}, \mathrm{n}(\%)$ & $12(15)$ \\
\hline Abdominal obesity,n (\%) & $12(15)$ \\
\hline Total fat $(\mathrm{kg})$,median $( \pm \mathrm{SE})$ & $11,36(8,9)$ \\
\hline Total Lean (kg),median ( \pm SD) & $26,1(8,9)$ \\
\hline Total mass $(\mathrm{kg})$,median $( \pm \mathrm{SD})$ & $38,8(16,7)$ \\
\hline Trunk fat/total fat, median ( \pm SD) & $0,42(0,5)$ \\
\hline Legs fat/ total fat, median $( \pm \mathrm{SD})$ & $0,42(0,44)$ \\
\hline FMI $(\%)$ median $( \pm S D)$ & $7,5(5,3)$ \\
\hline FFMI (\%) median ( \pm SD) & $17,8(3,8)$ \\
\hline
\end{tabular}

Conclusions: In JIA patients, there is a linear relationship between FMI and disease activity measured by JADAS27, but most patients had a normal BMI. The establishment of this relationship (fat-inflammatory activity) would be transcendental due to the need to optimize the recommendations in the JIA approach. References:

[1] Alvarez-Nemecye J, Buenfil-Rello FA, Pacheco Pantoja El. Association between body composition and disease activity in rheumatoid arthritis. A sistematic review. Reumatol Clin. 2016;12(4):190-5.

Disclosure of Interest: None declared

DOI: 10.1136/annrheumdis-2017-eular.6014

\section{THU0490 MULTICENTRE STUDY OF LUPUS NEPHRITIS URINARY BIOMARKERS IN ADULT AND PAEDIATRIC PATIENTS}

P. Costa Reis ${ }^{1,2}$, K. Maurer ${ }^{2}$, L. Schanberg ${ }^{3}$, J.M. Burnham ${ }^{2}$, E. von Scheven ${ }^{4}$, K. O'Neil ${ }^{5}$, M. Klein Gitelman ${ }^{6}$, M. Petri ${ }^{7}$, K.E. Sullivan ${ }^{2} .{ }^{1}$ Hospital de Santa Maria, Lisbon, Portugal; ${ }^{2}$ The Children's Hospital of Philadelphia, Philadelphia; ${ }^{3}$ Duke University, Durnham; ${ }^{4}$ University of California San Francisco, San Francisco; ${ }^{5}$ Indiana University, Indianapolis; ${ }^{6}$ Children's Hospital of Chicago, Chicago; ${ }^{7}$ Johns Hopkins University, Baltimore, United States

Background: Glomerulonephritis is an important cause of morbidity and mortality in patients with systemic lupus erythematosus, particularly in children. Presently, renal histology is used for the diagnosis of lupus nephritis $(\mathrm{LN})$, but it only offers a description of a confined area of the kidney and is seldom repeated for monitoring due to its invasiveness. Urinary biomarkers that reflect $\mathrm{LN}$ activity and have prognostic value are much needed to guide the judicious use of immunosuppressive drugs in these patients.

We focused on three potential LN biomarkers: HER2, TWEAK and VCAM1.

HER2 (Human Epidermal Growth Factor Receptor 2) controls cell proliferation by regulating the intracellular levels of miR-26a and miR-30b, which inhibit the expression of cell-cycle related genes. HER2 was recently shown to be dramatically overexpressed in the kidneys of LN patients and in NZM2410 mice. TWEAK (TNF-related weak inducer of apoptosis) is involved in pro-inflammatory responses, angiogenesis and fibrosis. TWEAK is increased in the kidneys of LN patients.

VCAM1 (vascular cell adhesion molecule 1) participates in the acute phase of inflammation, controlling leukocyte infiltration. Urinary VCAM1 has emerged as a reliable indicator of the $L N$ activity index.

Objectives: To determine the clinical value of urinary HER2, TWEAK and VCAM1 as biomarkers of LN activity.

Methods: A multicentre study is being performed in an adult and paediatric cohort. All patients have biopsy-proven LN. Clinical data and urine samples are being collected in each visit. Controls are age and sex matched individuals without renal disease. Flares are identified with the SELENA-SLEDAI flare index. Urine supernatants are analysed by commercial enzyme-immunoassays for HER2, TWEAK and VCAM1.

Results: Currently, we have studied 83 LN paediatric patients and 38 controls. 
The majority are female $(85 \%)$. The average age is $15 \pm 2.5$ years. This cohort includes Hispanic (36\%), Asian (29\%), African-American (21\%) and Caucasian $(11 \%)$ children. LN class III or IV was diagnosed in $67 \%$ of patients. Nearly all were treated with hydroxychloroquine and steroids $(95 \%)$. Other drugs used include mycophenolate mofetil $(85 \%)$, cyclophosphamide $(43 \%)$, rituximab $(27 \%)$ and tacrolimus (2\%).

A significant increase in urinary HER2, TWEAK and VCAM1 levels was found in LN patients ( $p=0.005 ; p=0.006 ; p=0.01$, respectively) when compared to controls. HER2 levels reflected disease activity, increasing during flares.

In an adult cohort of $\mathrm{LN}$ patients $(\mathrm{N}=126)$ composed mainly of females $(80 \%)$ with an average age of $46 \pm 13$ years, we also found a significant increase of urinary HER2 when compared to controls $(p=0.002)$.

A strong correlation between the urinary levels of HER2, TWEAK and VCAM1 was not found.

Conclusions: Urinary HER2, TWEAK and VCAM1 were significantly increased in a paediatric cohort of $L N$ patients. In addition, significantly higher urinary HER2 levels were also found in an adult $L N$ cohort. This on-going study will further evaluate if these urinary markers, alone or in combination, can reflect disease activity and predict renal flares, analysing their potential value in clinical practice. References:

[1] PMIDs: 26016809; 22727560; 22788914.

Disclosure of Interest: None declared

DOI: 10.1136/annrheumdis-2017-eular.5223

\section{THU0491 COMPARISON OF CLINICAL AND SEROLOGICAL FEATURES OF JUVENILE AND ADULT-ONSET NEUROPSYCHIATRIC LUPUS IN SPANISH PATIENTS}

S. Garrote Corral, A. García Fernández, W.A. Sifuentes Giraldo, A.L. Boteanu, M.L. Gámir Gámir, A. Zea Mendoza. Rheumatology, Ramon y Cajal University Hospital, Madrid, Spain

Background: Neuropsychiatric (NP) manifestations are a main cause of morbidity and mortality in juvenile-onset systemic lupus erythematosus (jSLE). Some studies suggest that they are more frequent and severe in jSLE than in adult-onset SLE (aSLE).

Objectives: To compare the clinical and serological profile of pediatric and adult patients with neuropsychiatric lupus (NPSLE) treated in a Spanish tertiary center. Methods: A retrospective study of patients with jSLE (age of onset: $0-18 \mathrm{y}$ ) and aSLE (age of onset: $>18 \mathrm{y}$ ) seen in our center during the period 1988-2016 was performed. Case definitions of the American College of Rheumatology were used to identify NPSLE manifestations. Demographics, clinical and serological data were obtained through a review of their medical records.

Results: A total of 69 patients with NPSLE were included, aSLE 41 (59\%) and jSLE $28(41 \%)$, the comparison of groups is presented in the table. Most of them were Caucasian (92\%), mean age at diagnosis in adults was 36.4 years (range: 19-68) and 13.9 years (range: $8-18$ ) in children. The proportion of males was higher in the latter group. The mean duration of the disease was significantly greater in adults, as well as the time from SLE diagnosis to NP manifestation onset, although without significant difference. Central NP manifestations were the most frequent in both groups (aSLE 93\%, jSLE 96\%) regarding to the peripheral manifestations (aSLE 12\%, jSLE 11\%). The most frequent manifestations in aSLE were headache $(29 \%)$, cerebrovascular disease $(27 \%)$, seizures $(17 \%)$ and myelopathy $(15 \%)$, whereas in jSLE were seizures $(46 \%)$, headache $(29 \%)$, mood disorder/depression $(25 \%)$, psychosis $(18 \%)$ and autonomic disorders $(18 \%)$. A significant group of patients presented $\geq 2$ central manifestation during their evolution (aSLE 32\%, jSLE 41\%), with the mean number of manifestations in adults being 1.36 (range: $1-3$ ) and in children 1.44 Range: 1-4). Patients with jSLE developed lupus nephritis (LN) with a significantly higher frequency, as well as higher titres of anti-DNA antibodies, erythrocyte sedimentation rate (ESR) and hypocomplementemia. During the study period there was mortality in 2 cases of aSLE and 2 jSLE (5\% and 7\%, respectively).

\begin{tabular}{lccc}
\hline & Juvenile NPSLE & Adult NPSLE & p-value \\
\hline No. of patients & $28(41 \%)$ & $41(59 \%)$ & - \\
Women:men & $20: 8$ & $39: 2$ & $0.0060^{*}$ \\
Time of disease (months) & 19.8 & 232.5 & $0.0001^{*}$ \\
NP manifestations at onset & $7(25 \%)$ & $11(27 \%)$ & 0.8651 \\
Time from diagnosis to NP manifestation (months) & 42.4 & 87.1 & 0.1268 \\
Lupus nephritis & $16(57 \%)$ & $9(22 \%)$ & $0.0028^{*}$ \\
Antiphospholipid syndrome & $5(18 \%)$ & $10(24 \%)$ & 0.5182 \\
ANA $\geq 1 / 1280$ & $8(29 \%)$ & $11(27 \%)$ & 0.8736 \\
Anti-DNA ab (IU/ml) & 178.9 & 39.4 & $0.0005^{*}$ \\
Anti-Ro/SSA ab & $10(36 \%)$ & $17(41 \%)$ & 0.6308 \\
Anticardiolipin ab & $4(14 \%)$ & $10(24 \%)$ & 0.3054 \\
Anti-B2 glycopotein I ab & $5(18 \%)$ & $7(17 \%)$ & 0.9328 \\
Lupus anticoagulant & $8(29 \%)$ & $10(24 \%)$ & 0.6977 \\
Cryoglobulins & $6(21 \%)$ & $3(7 \%)$ & 0.0874 \\
ESR (mm/h) & 53.8 & 35.7 & $0.0199^{*}$ \\
CRP mg/l & 4.6 & 4.7 & 0.9687 \\
C3 low $(<80 \mathrm{mg} / \mathrm{dl})$ & $22(79 \%)$ & $16(39 \%)$ & $0.0012^{*}$ \\
C4 low $(<16 \mathrm{mg} / \mathrm{dl})$ & $22(79 \%)$ & $13(32 \%)$ & $0.0001^{*}$ \\
\hline
\end{tabular}

Conclusions: Our results corroborate that juvenile patients with NPSLE present higher disease activity compared to adults. There was no significant diference in the time from SLE diagnosis to NP manifestation onset, but tended to be shorter in jSLE. The spectrum of NPSLE was varied both groups and an important proportion developed $\geq 2$ manifestation. Mortality continues to be important in NPSLE in both age groups.

Disclosure of Interest: None declared

DOI: 10.1136/annrheumdis-2017-eular.3425

\section{THU0492 MACROPHAGE ACTIVATION SYNDROME AS THE INITIAL MANIFESTATION OF JUVENILE SYSTEMIC LUPUS ERYTHEMATOSUS}

S. Sato, Y. Uejima, E. Suganuma, T. Takano, S. Fujinaga, Y. Kawano. Saitama Children's Medical Center, Saitama, Japan

Background: Macrophage activation syndrome (MAS) is a severe, potentially life-threatening complication of autoimmune diseases in children. Little is known about the association between MAS and the onset of juvenile systemic lupus erythematosus (jSLE).

Objectives: The aim of this study was to determine the frequency and clinical features of MAS as the initial complication of jSLE.

Methods: During 2004 and 2016, we retrospectively reviewed the clinical and laboratory features of 46 jSLE patients diagnosed at the Saitama Children's Medical Center. Patients who were complicated with MAS at the same time as jSLE. These patients were compared with a control group composed of jSLE patients without MAS. The MAS was diagnosed according to preliminary guidelines.

Results: Fifteen patients (32.6\%) developed MAS during the initial stage of jSLE. Fever, leukopenia, thrombocytopenia, hyperferritinemia hypofibrinogenemia, increased aspartate aminotransferase (AST), and increased lactate dehydrogenase $(\mathrm{LDH})$ were more frequently observed in patients having jSLE with MAS than in those having jSLE without MAS. No differences were observed in serum C3 and C4 levels, or erythrocyte sedimentation rate (ESR) $(P<0.05)$. Especially, Seven patients $(46.7 \%)$ had neurologic symptoms that were significantly higher in those with MAS $(P<0.01)$. All patients received corticosteroids when jSLE with MAS diagnosis was established, among whom seven received pulse methylprednisolone therapy. Two patients were treated with IVIG. Nine patients with MAS were treated with immunosuppressants, including cyclophosphamide and mycophenolate mofetil, azathioprine. No patient involved in this study died.

Conclusions: MAS can be the initial manifestation of jSLE. MAS may be an underrecognized complication of jSLE. MAS with jSLE should be suspected in patients with high fever, cytopenia, and a liver disorder. In addition, we found that in jSLE with MAS patients, they had more neurologic symptoms compared to jSLE without MAS. Early diagnosis and intensive therapy is essential to improve the clinical outcome.

References:

[1] Parodi A, Davì S, Pringe AB, Pistorio A, Ruperto N, et al. Macrophage activation syndrome in juvenile systemic lupus erythematosus: a multinational multicenter study of thirty-eight patients. Arthritis Rheum. 2009 Nov;60(11):3388-99.

[2] Pringe A, Trail L, Ruperto N, Buoncompagni A, Loy A, et al. Macrophage activation syndrome in juvenile systemic lupus erythematosus: an underrecognized complication? Lupus. 2007;16(8):587-92.

[3] Gormezano NW, Otsuzi Cl, Barros DL, da Silva MA, Pereira RM, et al. Macrophage activation syndrome: A severe and frequent manifestation of acute pancreatitis in 362 childhood-onset compared to 1830 adult-onset systemic lupus erythematosus patients. Semin Arthritis Rheum. 2016 Jun;45(6):706-10.

Disclosure of Interest: None declared

DOI: 10.1136/annrheumdis-2017-eular.5935

\section{THU0493 STUDY OF LONG-TERM OUTCOME OF CHILDREN WITH JUVENILE DERMATOMYOSITIS FROM A SINGLE-CENTRE IN NORTH INDIA}

A. Sharma, A. Gupta, A. Rawat, D. Suri, S. Singh. Pediatrics, Post Graduate Institute of Medical Education and Research, Chandigarh, India, Chandigarh, India

Background: Juvenile dermatomyositis (JDM) is a rare inflammatory myopathy seen in children. There have been few studies on long-term outcome in children with JDM

Objectives: To assess long-term outcome of JDM using validated measures of outcome

Methods: All children diagnosed to have JDM for more than 2 years and registered in Pediatric Rheumatology Clinic at PGIMER, Chandigarh, India, were deemed eligible for recruitment. Study period was from January 1, 2015 to June 30,2016 . Those who were not on regular follow-up were called for assessment which was done by a single observer using Childhood Myositis Assessment Scale (CMAS), Manual Muscle Testing 8 (MMT8), Myositis Disease Activity Assessment Tool (MDAAT), abbreviated cutaneous assessment tool (aCAT), Myositis Damage Index (MDI) and Childhood Health Assessment Questionnaire (CHAQ).

Results: Thirty-five children were enrolled in this study, $22(62.9 \%)$ were on regular follow-up. Mean age was 13.9yrs (range 4-29yrs). Mean age at diagnosis was $7.51 y r s$ with median interval between onset of symptoms and diagnosis 